\title{
Physical exercise reduces transplant arteriosclerosis in a mouse aorta transplantation model
}

\author{
Wiebke Sommer, MD, ${ }^{\mathrm{a}}$ Ann-Kathrin Knöfel, PhD, ${ }^{\mathrm{a}}$ Nicole Izykowski, MSc, ${ }^{\mathrm{b}}$ Felix Oldhafer, MD, ${ }^{\mathrm{a}}$ \\ Murat Avsar, MD, ${ }^{\mathrm{a}}$ Danny Jonigk, MD, ${ }^{\mathrm{b}}$ Gregor Warnecke, MD, ${ }^{\mathrm{a}}$ and Axel Haverich, MD
}

Background: Transplant arteriosclerosis limits long-term outcome after heart transplantation. The underlying mechanism of transplant arteriosclerosis remains alloreactivity, but it is also influenced by nonimmunologic cofactors. Physical exercise has well-established effects on the prevention of native arteriosclerosis. We hypothesized that physical exercise would reduce the development of transplant arteriosclerosis in an allogeneic transplantation setting.

Methods: Segments of the thoracic aorta from C57.B16 (H2b) or C3H.HeJ (H2k) mice were transplanted into the abdominal aortas of CBA.Ca mice (H2k), representing a major or minor alloantigen mismatch, respectively. Three days after surgery, recipient mice were assigned to either the control or physical exercise (consisting of $2 \times 45$ minutes of treadmill training per day) groups. Transplant arteriosclerosis was assessed and quantified by histology on day 28 after vessel transplantation. Endothelial cell integrity and function in histology sections and peripheral blood was assessed.

Results: All animals developed transplant arteriosclerosis with more severe luminal occlusion in the major alloantigen mismatch setting. Animals undergoing physical exercise developed significantly less severe transplant arteriosclerosis in both the major $(P<.0001)$ and minor $(P<.0001)$ antigen mismatches than their respective control groups without physical exercise. $\mathrm{CD} 31^{+}$endothelial cells were present in significantly higher numbers in the grafts and circulating in peripheral blood in the exercise groups compared with their respective control. Above that, we found enhanced endothelial nitric oxide synthase-positive cells in both exercise groups compared with the untreated groups ( $P=.01$ and $P=.02$, respectively).

Conclusions: Physical exercise has a protective effect against the development of transplant arteriosclerosis. This could be due to enhanced endothelial cell regeneration and function in the graft. (J Thorac Cardiovasc Surg 2015;149:330-7)

See related commentary on pages 337-9.

Cardiac transplantation is the gold standard therapy for end-stage heart failure. However, chronic rejection, which is known as cardiac allograft vasculopathy (CAV), in transplanted hearts, affects in excess of $50 \%$ of the recipients at 10 years. ${ }^{1}$ CAV thus remains the most important cause of allograft loss in heart transplantation. The pathology comprises an occlusive disease of the coronary arteries,

From the Division of Cardiac, Thoracic, Transplantation, and Vascular Surgery and the Institute of Pathology, ${ }^{\mathrm{b}}$ Hannover Medical School, Hanover, Germany.

Supported by grants from the Deutsche Forschungsgemeinschaft (SFB 738).

Disclosures: Authors have nothing to disclose with regard to commercial support.

W.S. and A.-K.K. contributed equally to this work.

G.W. and A.H. share last authorship.

W.S., N.I., D.J., G.W., and A.H. are members of the German Center for Lung Research.

Received for publication Aug 2, 2014; revisions received Oct 1, 2014; accepted for publication Oct 5, 2014.

Address for reprints: Wiebke Sommer, MD, Division of Cardiac, Thoracic, Transplantation, and Vascular Surgery, Hannover Medical School, Carl-Neuberg-Str.1, 30623 Hannover, Germany (E-mail: sommer.wiebke@mh-hannover.de).

$0022-5223 / \$ 36.00$

Copyright (C) 2015 by The American Association for Thoracic Surgery http://dx.doi.org/10.1016/j.jtcvs.2014.10.029 with similarities to atherosclerotic coronary artery disease (CAD) in patients who do not undergo transplant. CAV develops in both epicardial and intramural coronary vessels and may show concentric intima and media thickening. ${ }^{2,3}$ Progression of CAV takes place in the absence of arteriosclerosis in other recipient arteries, indicating allogeneic immune responses as the underlying trigger. However several other factors have been identified to also contribute to progress of CAV. Kobashigawa and colleagues ${ }^{4}$ described the effectiveness of early statin therapy in heart transplant recipients, leading to significantly lower serum cholesterol levels, better 1-year survival, and lower incidence of CAV. Also, cytomegalovirus serostatus positivity as well as episodes of cytomegalovirus infection influence development and progression of $\mathrm{CAV}^{5}$

More recently, the endovascular endothelium and its microvascularization has been the focus of CAV research, indicating that pathologic coronary artery microcirculation is a negative prognostic factor for long-term outcome after cardiac transplantation. Therefore, impaired endovascular microcirculation possibly influences the onset of CAV in allografts. ${ }^{6}$ The degree of microvascularization in vessels might also be influenced by physical exercise, given that for muscle, as well as Achilles tendon tissue, microvascular 

Abbreviations and Acronyms
$\mathrm{CAD}=$ coronary artery disease
$\mathrm{CAV}=$ cardiac allograft vasculopathy
cDNA $=$ complementary DNA
eNOS $=$ endothelial nitric oxide synthases
$\mathrm{EPC}=$ endothelial progenitor cell
$\mathrm{PCR}=$ polymerase chain reaction

filtration is enhanced under physical exercise. ${ }^{7,8}$ Another beneficial effect of physical exercise in recipients of heart transplant was described by Hermann and colleagues, ${ }^{9}$ who showed reduced systolic blood pressures and improved endothelial function as determined in duplex sonography of nitroglycerin-induced vasodilation. Direct experimental evidence supporting the observation of reduced transplant arteriosclerosis by physical exercise is currently missing.

Here, we hypothesize physical exercise directly counteracts the development of transplant arteriosclerosis in a murine allogeneic aorta transplantation model.

\section{MATERIAL AND METHODS}

Mice

C57.B16 mice (H2b), CBA.Ca (H2k) and C3H.HeJ (H2k) mice were obtained from Charles River or Jackson Laboratories (Wilmington, Mass) and housed under specific pathogen-free conditions. The mice were aged 8 to 12 weeks at the time of surgery. All animals received humane care in compliance with the German animal protection legislation, the Principles of Laboratory Animal Care, and the Guide for the Care and Use of Laboratory Animals. ${ }^{10}$ The study was approved by the government board for animal welfare of Lower Saxony, Germany.

\section{Surgical Technique}

Transplantation of aorta grafts from C57.B16 (H2b), C3H.HeJ (H2k), or CBA.Ca (H2k) donor mice into CBA.Ca $(\mathrm{H} 2 \mathrm{k})$ recipient mice was performed using a technique described by Koulack and colleagues. ${ }^{11}$ Briefly, the thoracic aorta of the donor mice was explanted, flushed with heparinized sodium chloride, and stored at $4^{\circ} \mathrm{C}$. Grafts of 3 to $4 \mathrm{~mm}$ length were then transplanted into the abdominal aorta of the recipient animals (Figure 1, A).

\section{Physical Exercise}

Animals undergoing physical exercise were kept in regular cages, but were put into a cage with a treadmill twice daily for 45 minutes training per session. Training commenced as of postoperative day 3 after recovery from surgery. The physical exercise program was conducted until postoperative day 28 (Figure $1, B$ and $C$ ).

\section{Experimental Groups}

To resemble a full major alloantigen mismatch, C57.B16 (H2b) donor aortas were transplanted into CBA.Ca $(\mathrm{H} 2 \mathrm{k})$ recipient mice. A group of 10 recipients received no further treatment and constituted the control group, whereas another 10 recipients performed the treadmill training program described above. A second set of experiments was set up in a minor alloantigen mismatch, transplanting $\mathrm{C} 3 \mathrm{H} . \mathrm{HeJ}(\mathrm{H} 2 \mathrm{k})$ vessels into CBA.Ca $(\mathrm{H} 2 \mathrm{k})$ mice. A group of 11 recipients received no further treatment and constituted the control group, whereas another 11 recipients performed the treadmill training program. Further, 2 control groups of CBA.Ca
(H2k) mice undergoing sham surgery without aorta transplantation but performing treadmill training (CBA.Ca $[\mathrm{H} 2 \mathrm{k}]$; that is, the sham exercise group; $\mathrm{n}=4$ ) or not (CBA.Ca $[\mathrm{H} 2 \mathrm{k}]$; that is, the sham control group; $\mathrm{n}=4$ ), were analyzed and served as sham groups. Additionally, 3 CBA.Ca $(\mathrm{H} 2 \mathrm{k})$ animals $(\mathrm{n}=3)$ underwent aorta transplantation receiving isogeneic grafts from CBA.Ca (H2k) mice. The experimental setup is shown in Figure 1.

\section{Flow Cytometry}

Circulating endothelial cells in peripheral blood of recipient mice were monitored by flow cytometry. Whole blood samples were collected into Eppendorf tubes and stained with antimurine CD31 antibody (Biolegend, San Diego, Calif). Analyses were performed on a FACSCanto flow cytometer (Becton, Dickinson and Co, Franklin Lakes, NJ). Obtained data were analyzed using FACSDiva 6.3.1 software (Becton, Dickinson, and Co).

\section{Analyses of the Aorta Grafts}

Transplanted aorta grafts were harvested on postoperative day 28 . The grafts were then flushed with heparinized saline and imbedded into paraffin. Sections of 2- to 3- $\mu \mathrm{m}$ thickness were cut after complete removal of the anastomosis area and stained with hematoxylin and eosin or van Giesson elastin stain. Morphometric analysis of the graft was performed on 3 different van Giesson elastin-stained cuts, taken on 3 different levels being apart a minimum of 10 microtome cuts per aorta. Histologic photographs were taken at $\times 40$ magnification using a light microscope connected to a digital camera (Olympus U-CMAD3, Olympus, Hamburg, Germany). Digitized images were analyzed using Photoshop CS4 software (version 11.0.2, Adobe, San Jose, Calif). Briefly, the endoluminal area, as well as the areas within the internal and external laminae elasticae, were circumscribed and the absolute pixel counts of each area was then recorded. From these measurements, the quotient for the thickness of the intima; that is, the intima to media ratio, was calculated. This ratio indicates relative thickness $(\%)$ of the intima $(R=$ [area (intima)/area (media) $] \times 100 \%)$. For each individual experiment a mean value of the 3 sections analyzed was calculated.

\section{Immunohistochemistry}

Histologic sections of $2-\mu \mathrm{m}$ thickness were deparaffinized and incubated with primary polyclonal rabbit against mouse endothelial nitric oxide synthases (eNOS) (Abcam, UK) or polyclonal rabbit CD31 (Santa Cruz Biotechnology, Heidelberg, Germany) antibodies over night at $4^{\circ} \mathrm{C}$. Then, 1-step polymer antirabbit (Zytomed Systems GmbH, Berlin, Germany) was applied. To visualize the staining, the sections were incubated with dimethylaminoazobenzene (DAB-Komplex, Zytomed Systems GmbH, Berlin, Germany) and counterstained with hematoxylin (C. Roth GmbH, Karlsruhe, Germany). For further analyses, histologic sections were clustered into fields of identical size. Subsequently, the number of positive cells per field was counted.

\section{Polymerase Chain Reaction (PCR)}

For RNA extraction and complementary DNA (cDNA) preparation, formalin-fixed and paraffin-embedded tissue was cut. Tissue was suspended overnight in a proteinase $\mathrm{K}$ digestion solution. RNA was isolated using phenol-chloroform extraction followed by ethanol precipitation. Then, $10 \mu \mathrm{L}$ RNA were transcribed into cDNA using the High Capacity cDNA Reverse Transcription Kit (Applied Biosystems, Darmstadt, Germany) following the manufacturer's instructions. To increase the sensitivity of the subsequent real-time PCR analysis several thousand-fold, the cDNA was preamplified in 14 PCR cycles with nonrandom PCR primers (PreAmp Master Mix Kit, Applied Biosystems Darmstadt, Germany). ${ }^{12}$ For analyzing the messenger RNA expression, the preamplified cDNA was evaluated by real-time PCR (TaqMAn 7500 Real-Time PCR System, Applied Biosystems, Carlsbad, Calif). Quantification was performed in 


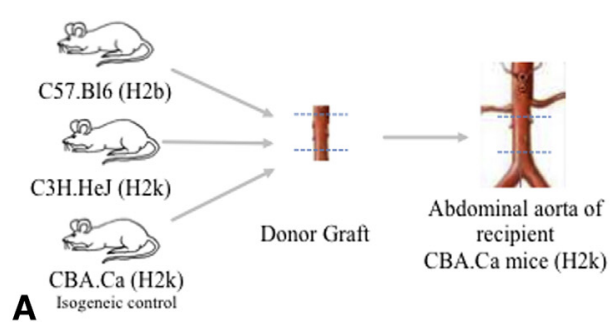

A Isogeneic control
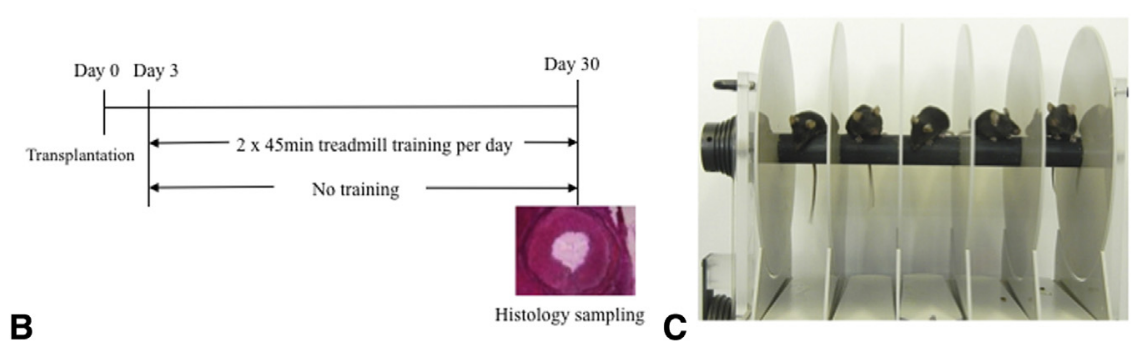

FIGURE 1. A, Schematic setup of the aorta transplantation model. Aorta segments from C57.B16 (H2b) (major mismatch), C3H-HeJ (H2k) (minor mismatch), or CBA.Ca (H2k) (isogeneic controls) donor mice were transplanted into the abdominal aorta of CBA.Ca (H2k) recipient mice. B, Workflow scheme for treadmill training or no training and histologic sampling on day 30 after transplantation. C, Mice undergoing treadmill training twice daily for 45 minutes.

reactions containing preamplified cDNA, TaqMan gene Expression Master Mix and the individual TaqMan Gene Expression Assay (both from Applied Biosystems). The following genes were analyzed: eNOS (NOS3) (Mm00435217_m1, 71 bp) and 3 housekeeping genes were used GusB (Mm00446953_m1, 76 bp), HPRT (Mm00446968_m1, 65 bp), and Polyr2 $\alpha$ (Mm00839502_m1, 70 bp). For negative control, cDNA was replaced by water. Gene expression values were converted to relative expression using the $\triangle \mathrm{CT}$ method by normalization to the mean expression of the three housekeeping genes (GusB, HPRT, and Polyr $2 \alpha)$ and the relative expression was calculated as 2- $\Delta$ CT with Excel (Microsoft, Redmond, Wash).

\section{Statistical Analysis}

Obtained data were analyzed using the Mann-Whitney $U$ test or 1-way analysis of variance followed by Bonferroni's post hoc testing, as appropriate. All data were reported as means \pm standard error of the mean. For statistical analyses, GraphPad Prism version 5.0 for Windows (GraphPad Software, San Diego, Calif) was used. The group size number in the treadmill training and control groups for comparison of the degree of transplant arteriosclerosis developed, was chosen at a minimum of $\mathrm{n}=10$, to allow for parametric testing. In some additional experiments, such as sham controls, smaller group sizes were accepted and analyzed by nonparametric testing.

\section{RESULTS}

All animals survived the overall observation period of 28 days after aorta transplantation.

\section{Physical Exercise Reduces Transplant \\ Arteriosclerosis in Allogeneic Transplantation}

Donor aorta segments from C57.B16 (H2b) mice were transplanted into untreated fully allogeneic CBA.Ca (H2k) recipient mice. After 28 days, transplant arteriosclerosis caused occlusion of $81.1 \% \pm 3.6 \%$ of the donor aorta lumen. Mice undergoing treadmill training developed significantly less luminal occlusion $(60.4 \% \pm 4.1 \%$;
$P<.0001$ ) (Figure 2, $B$ and $E$ ). Bonferroni posttesting confirmed these results. A further mouse strain combination was set up where all major histocompatibility complex antigens were matched and only minor antigens mismatched. Aorta segments from C3H.HeJ (H2k) donor mice were transplanted into untreated CBA.Ca (h2k) recipient mice. In this setting luminal occlusion of the donor aorta amounted to $74.9 \% \pm 5.1 \%$. Again, this was significantly reduced in the group of animals undergoing treadmill training $(48.6 \% \pm 6.4 \% ; P<.001)$ (Figure 2, $C$ and $F$ ), Bonferroni posttesting approving the obtained results. In contrast, CBA.Ca (H2k) not undergoing aorta transplantation but performing treadmill training showed no luminal obstruction, as did CBA.Ca (H2k) mice without transplantation and no treadmill training $(3.7 \% \pm 1.4 \%$ vs $3.4 \%$ $\pm 1.2 \%$; not significant). Also, CBA.Ca (H2k) mice receiving isogeneic grafts from CBA.Ca $(\mathrm{H} 2 \mathrm{k})$ mice did not develop significant luminal obstruction $(10.4 \% \pm$ $4.9 \%$ ) (Figure 2, $A$ and $D$ ).

\section{Physical Exercise Increases Circulating Endothelial Cell Numbers}

Frequencies of circulating endothelial cells expressing CD31 were measured in peripheral blood mononuclear cells by flow cytometry. Mice undergoing treadmill training showed significantly higher frequencies of $\mathrm{CD} 31^{+}$cells in peripheral blood than control animals in both the major $(49.7 \% \pm 8.2 \%$ vs $22.6 \% \pm 4.8 \% ; P=.01)$ and minor alloantigen mismatch settings $(31.3 \% \pm 4.9 \%$ vs $18.5 \%$ $\pm 2.8 \% ; P=.04$ ) (Figure $3, B$ and $C$ ). In contrast, the control cohorts without aorta transplantation show no significant difference in the number of cells expressing 
CBA.Ca (H2k) $\rightarrow$ CBA.Ca (H2k)

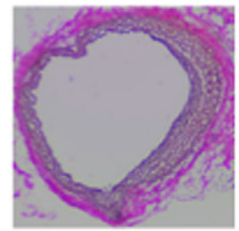

Isogeneic aorta
C57.B16 (H2b) $\rightarrow$ CBA.Ca $(\mathrm{H} 2 \mathrm{k})$

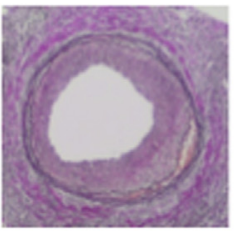

exercise group

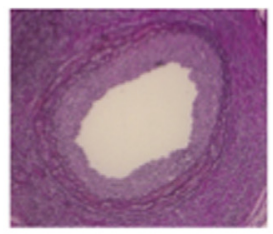

control group
C3H.HeJ (H2k) $\rightarrow$ CBA.Ca (H2k)

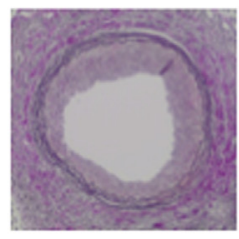

exercise group

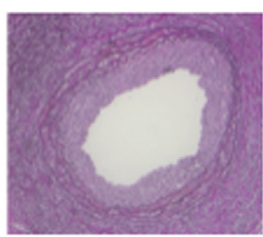

control group
A

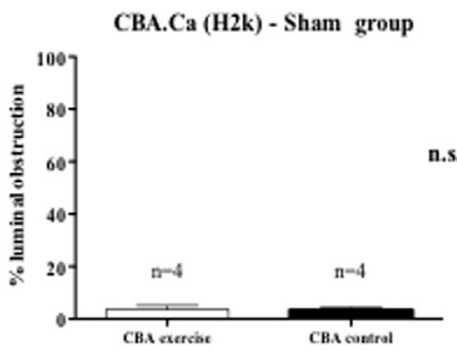

B

C
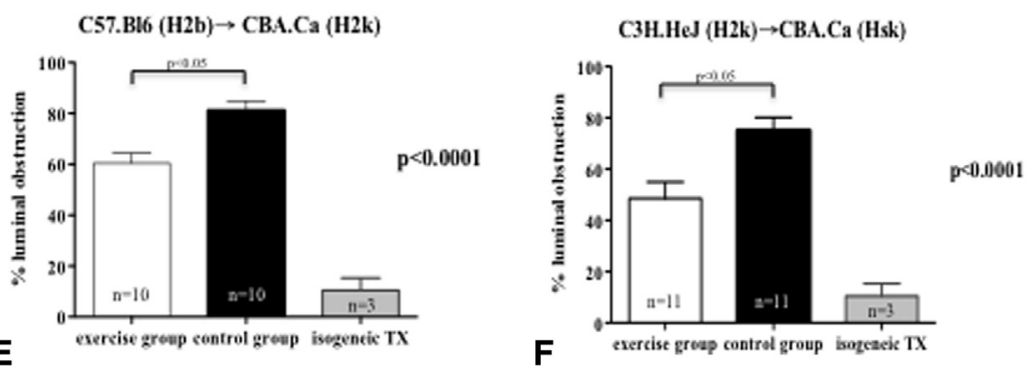

FIGURE 2. A, Histology of isogeneic aorta transplants on day 30 after transplantation (CBA.Ca [H2k] donor and recipient). No significant transplant arteriosclerosis is detectable. (van Giessen elastin staining; $\times 40$ magnification.) B, Histology of aorta grafts on day 30 after transplantation. C57.B156 (H2b) donor mice into CBA.Ca (H2k) recipients representing a major alloantigen mismatch. (van Giessen elastin staining; $\times 40$ magnification.) C, Histology of aorta grafts on day 30 after transplantation. C3H-HeJ (H2k) donor mice into CBA.Ca (H2k) recipients representing a minor alloantigen mismatch. (van Giessen elastin staining; $\times 40$ magnification.) D, Luminal obstruction of animals not undergoing aorta transplantation but performing treadmill training or not (CBA exercise/control sham group). No significant luminal obstruction is detectable in either group. E, Luminal obstruction in the major alloantigen mismatch group (C57.B156 [H2b] donors and CBA.Ca [H2k] recipients). Significantly less luminal obstruction is detectable in the group undergoing treadmill training (Bonferroni post hoc test, control vs exercise group; $P<.05$ ), in comparison with animals receiving major allogeneic aorta transplantation and animals receiving isogeneic aorta transplantation (1-way analysis of variance; $P<.0001$ ). F, Luminal obstruction in minor alloantigeneic mismatch group (C3H-HeJ [H2k] donor mice into CBA.Ca [H2k] recipient mice). Significantly less luminal obstruction is detectable in the group undergoing treadmill training in comparison with animals not performing treadmill training (Bonferroni post hoc test; $P<.05$ ) and mice undergoing isogeneic aorta transplantation (1-way analysis of variance; $P<.0001)$. $T X$, Transplantation.

CD31 in peripheral blood between animals performing treadmill training or not $(56.1 \% \pm 4.3 \%$ vs $44.3 \% \pm$ $7.5 \%$; not significant) (Figure 3, A).

\section{Physical Exercise Improves Endothelial Cell Integrity in Grafts}

Donor aorta segments were harvested from recipient mice for histology 28 days after transplantation. Immunohistochemical staining for CD31 (Figure 4, B) revealed higher numbers of $\mathrm{CD} 31^{+}$cells per high power field in recipients of fully allogeneic grafts transplanted across a major alloantigen mismatch and undergoing treadmill training compared with controls without treadmill training, although no statistical significance was reached (Figure 4, $D$ ). Grafts harvested from recipients of minor alloantigen mismatched transplants generally expressed lower levels of CD31, indicating lower endothelial cell density. However, grafts from animals with treadmill training again had more $\mathrm{CD} 1^{+}$cells per high power field than the respective controls (Figure 4,E).

For comparison, mice without aorta transplantation showed highest expression of CD31+ cells, although those animals performing treadmill training also had significantly more CD31+ cells compared with those not performing training (Figure 4, $A$ and $C$ ).

\section{Physical Exercise Maintains Functional Integrity of Endothelial Cells}

eNOS is constitutively expressed in endothelial cells and has been linked to the function of healthy endothelium. Histologic sections from aorta grafts harvested at day 28 were stained using the anti-eNOS antibody ab5589 (Abcam). Endothelial cells staining positive for eNOS were then counted per high power field. In the major alloantigen mismatch strain combination, mice with treadmill training showed more $\mathrm{eNOS}^{+}$cells than the animals without treadmill training $\left(17.6 \pm 1.4 \mathrm{eNOS}^{+} /\right.$field vs $7.0 \pm 1.4 \mathrm{eNOS}^{+} /$field; $P=.0007$ ) (Figure 5, B). The respective difference in the minor alloantigen mismatch setting was $8.4 \pm 1.2 \mathrm{eNOS}^{+} /$field in the treadmill training group versus $5.4 \pm 0.2 \mathrm{eNOS}^{+} /$field in the control group $(P=.04)$ (Figure 5, $C$ ). Again, no significant difference in eNOS expression was detectable among control cohorts without aorta transplantation $(12.2 \pm 5.4 \mathrm{vs}$ $16.0 \pm 1.0 \mathrm{eNOS}^{+} /$field; not significant) (Figure 5, A). 


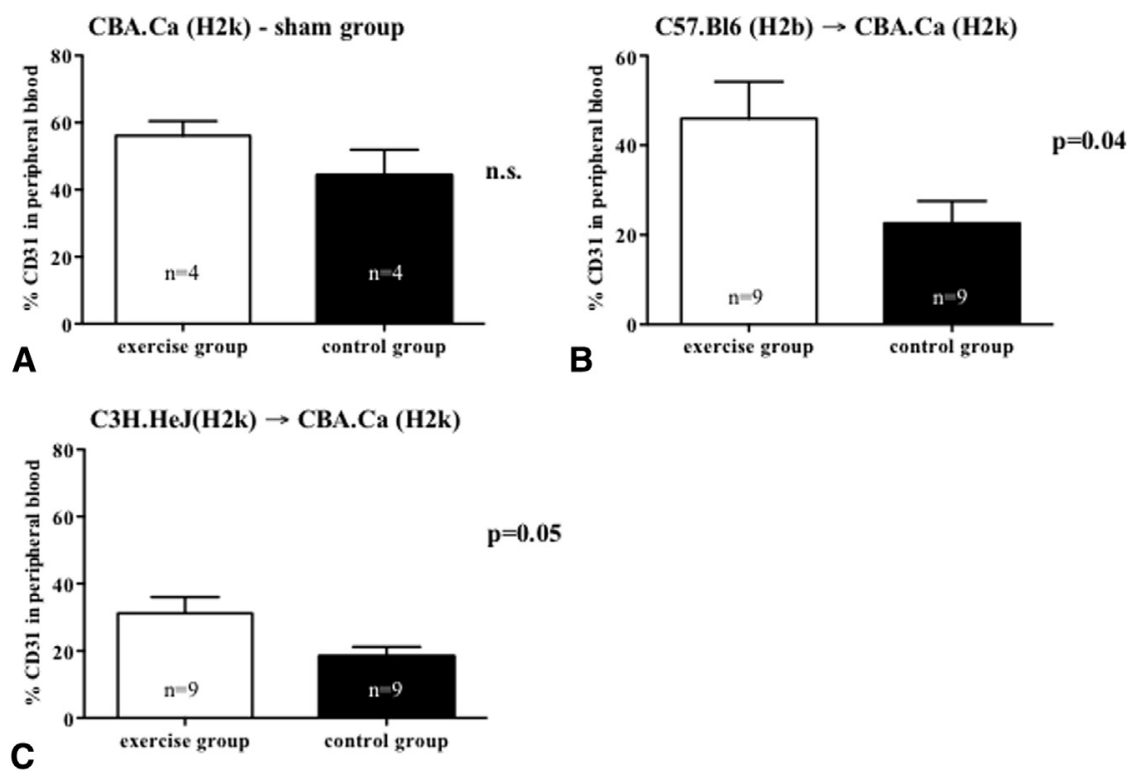

FIGURE 3. A, Percentage of $\mathrm{CD} 31^{+}$cells in peripheral blood in animals not undergoing aorta transplantation as detected by flow cytometry. No significant difference of $\mathrm{CD} 31^{+}$cells was detectable between animals performing treadmill training and animals not performing treadmill training (Mann-Whitney $U$ test; not significant). B, Percentage of $\mathrm{CD} 31^{+}$cells in peripheral blood in transplant recipient mice in the major alloantigeneic mismatch group (C57.B156 [H2b] donor mice into CBA.Ca [H2k]) as detected by flow cytometry. Significantly higher percentages of CD31 ${ }^{+}$cells in peripheral blood were traceable on day 30 in mice undergoing physical exercise compared with the no exercise group (Mann-Whitney $U$ test; $P=.04$ ). C, Percentage of $\mathrm{CD} 31^{+}$cells in peripheral blood in transplant recipient mice in the minor alloantigeneic mismatch group (C3H.HeJ [H2k] donor mice into CBA.Ca $[\mathrm{H} 2 \mathrm{k}]$ ) as detected by flow cytometry. Higher percentages of $\mathrm{CD} 31^{+}$cells in peripheral blood were traceable on day 30 in mice undergoing physical exercise as compared to the no exercise group, although not statistically significant (Mann-Whitney $U$ test; $P=.05$ ).

We then further studied eNOS gene expression in the grafts by PCR. In the major alloantigen mismatch setup a moderately augmented relative expression of eNOS was found in animals undergoing treadmill training compared with the control group; however, this difference did not reach statistical significance $(0.39 \pm 0.04$ vs $0.29 \pm 0.04$; not significant) (Figure 5, E). In the minor alloantigen mismatch groups, no significant difference in eNOS expression was found $(0.34 \pm 0.06$ vs $0.35 \pm 0.05 ; P=.91)$ (Figure 5, F). For comparison, mice not undergoing aorta transplantation revealed highest eNOS expression; however, no significant difference was detectable between animals performing treadmill training or not $(1.5 \pm 0.2$ vs $2.2 \pm 0.5$; not significant) (Figure 5, D).

\section{DISCUSSION}

Here, we show that physical exercise is an important modulator of the development of transplant arteriosclerosis in a defined experimental setting. Whereas the model of fully allogeneic aorta transplantation in untreated mice as used in our study invariably led to severe transplant arteriosclerosis after 28 days, an intensive treadmill training program significantly reduced the severity by more than $25 \%$. Further, we show that endothelial integrity and function is improved in animals undergoing treadmill training and this could be due to increased endothelial cell regeneration through bloodborne endothelial cell turnover. By performing the same exercise protocol in animals not undergoing aorta transplantation as well as in animals undergoing isogeneic aorta transplantation-both cohorts without developing luminal obstruction-the influence of allogeneic transplantation is evident.

Lack of physical exercise is a well-established clinical risk factor for arteriosclerosis of native arteries and especially CAD. Thus, conversely, physical exercise has been propagated to prevent the development of CAD. ${ }^{13-15}$ Given the phenotypic similarities between CAD and arteriosclerosis of the transplanted heart; that is, CAV, a comparable positive effect of physical exercise in the prevention of CAV was suggested. This connection as well as its mechanism has not been firmly established, partly because the positive effects of physical exercise on graft coronary arteries might be masked by other, potentially even more positive effects of physical exercise. Physical exercise overwhelmingly influences skeletal muscle function, cardiac function, heart rate, and quality of life in patients with $\mathrm{CAD},{ }^{14,15}$ but also with CAV. Therefore, physical exercise has been a longstanding therapeutic tool in treatment algorithms after heart transplantation. ${ }^{16,17}$ Only recently, a potential direct effect of physical exercise on the development of CAV was recognized. Nytrøen and colleagues ${ }^{18}$ reported on significantly reduced progress 

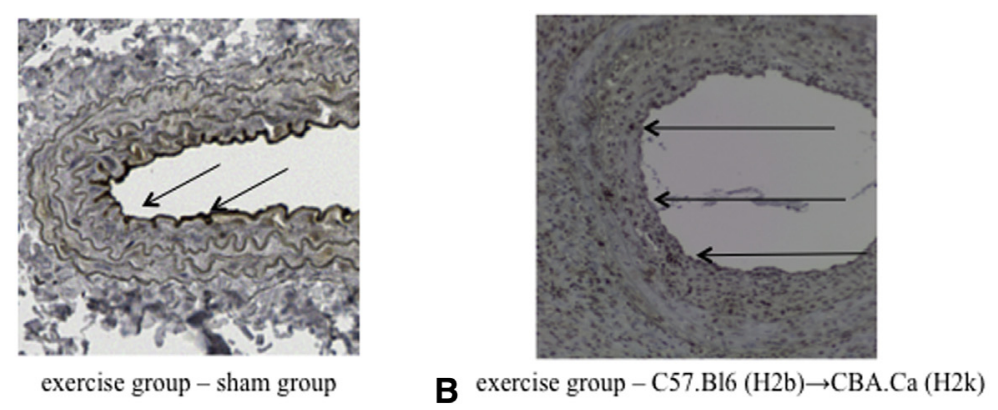

A
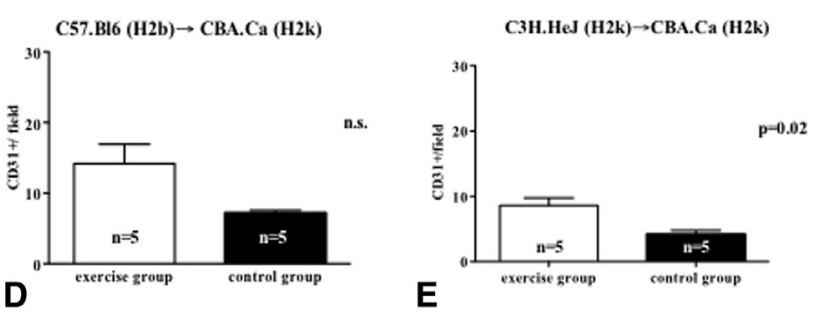

FIGURE 4. A, Immunohistologic staining of an aortic graft of the sham group not undergoing aorta transplantation detecting CD31+ cells (arrows). $\mathrm{B}$, Immunohistologic staining of an aortic graft of a CBA.Ca (H2k) mouse receiving a major alloantigeneic graft from a C57.B16 (H2b) animal performing treadmill training (exercise group), detecting CD $31^{+}$cells (arrows). C, Detection of CD31 $1^{+}$cells/field in immunohistologic staining of aortic grafts on day 30 after either treadmill training or no training (sham group). Animals undergoing daily physical exercise showed significantly higher numbers of CD $31^{+}$ cells/field compared with the control group (Mann-Whitney $U$ test; $P=.03$ ). D, Detection of CD $31^{+}$cells/field in immunohistologic staining of aortic grafts on day 30 after transplantation in the major alloantigeneic mismatch group. Animals undergoing physical exercise showed higher numbers of CD31 ${ }^{+}$cells/ field compared with the no exercise group, although results do not reach statistical significance (Mann-Whitney $U$ test; not significant). E, Detection of $\mathrm{CD} 31^{+}$cells/field in immunohistologic staining of aortic grafts on day 30 after transplantation in the minor alloantigeneic mismatch group. Animals undergoing physical exercise showed significantly higher numbers of $\mathrm{CD} 31^{+}$cells/field compared with the control group (Mann-Whitney $U$ test; $P=.02$ ).

of CAV in clinical heart transplantation recipients undergoing high-intensity interval training on a regular basis compared with a control group not performing physical exercise.

Along these lines a study in long-term heart transplant recipients performing high intensity physical exercise for 8 weeks revealed not only significantly reduced systolic blood pressure but also improved intravasal endothelial function compared with a control cohort. Endothelial function was measured by ultrasound diagnostic of the brachial artery diameter response to nitroglycerine, which was administered sublingually. ${ }^{9}$ In addition, maximal endovasal oxygen uptake as measured in the exhaled air was increased, potentially indicating a mechanism counteracting endothelial dysfunction, one of the proposed underlying mechanisms of CAV. ${ }^{19}$

Transplant arteriosclerosis occurs as a result of multiple immunologic and nonimmunologic injuries and risk factors, eventually resulting in intima hyperplasia and fibroelastic proliferation as well as outward remodeling of the graft adventitia, all leading to luminal stenosis. ${ }^{20,21}$ Nonimmunologic risk factors are the regulation of blood pressure, adequate control of normoglycemia, as well as lipid metabolism control. Although physical exercise, or rather the lack thereof, may have to be added to that nonimmunologic risk factor list soon, the mechanism of its influence on the development of transplant arteriosclerosis is less well understood.

Our data show that regular physical exercise reduces transplant vasculopathy in a murine aorta transplantation model. The degree of luminal obliteration of the transplanted aorta in mice undergoing regular treadmill training was significantly lower than observed in control animals not performing an exercise protocol. Because this effect was similarly observed in both the major and minor alloantigen mismatch settings, we exclude differential $M H C$ gene expression in the grafts as a potential mechanism causing the protective effect of physical exercise on the graft endothelium. Various parameters indicate a better preserved graft endothelium in the groups undergoing treadmill training. The underlying mechanisms of this observation were then investigated further; however, quality of endothelial function seems to play an important role in the development of transplant arteriosclerosis. Knowing that metabolism of endothelial cells is directly dependant on nitric oxide, which is synthesized from L-arginine by eNOS, ${ }^{22}$ a direct correlation between endothelial function and the existence of nitric oxide-linked vasodilative stimulation on the development of transplant vasculopathy is conceivable. Our results show that significantly less eNOS was detectable in the endothelium of the transplanted aortic grafts in the control groups not 

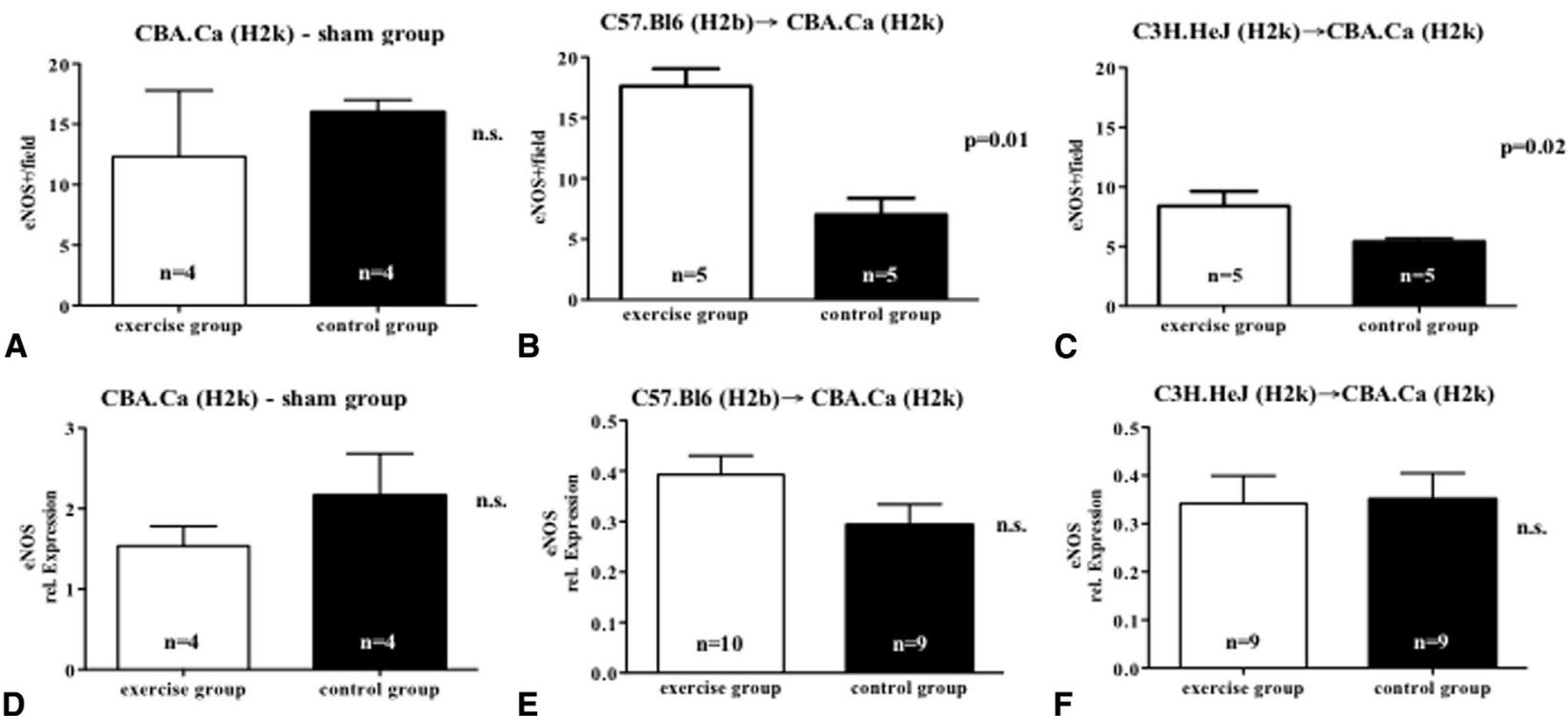

FIGURE 5. A, Analysis of immunohistologic detection of positive endothelial nitric oxide synthases $\left(e N O S^{+}\right)$cells/field in the sham group not receiving aorta transplantation. No significant difference between animals undergoing physical exercise and animals not performing exercise was detectable (MannWhitney $U$ test; not significant). B, Analysis of immunohistologic detection of eNOS ${ }^{+}$cells/field in the major alloantigeneic mismatch group. Animals undergoing physical exercise showed significantly higher numbers of $\mathrm{eNOS}^{+}$cells in the transplanted grafts compared with animals not performing exercise (Mann-Whitney $U$ test; $P=.01$ ). C, Analysis of immunohistologic detection of eNOS ${ }^{+}$cells/field in the minor alloantigeneic mismatch group. Animals undergoing physical exercise showed significantly higher numbers of $\mathrm{eNOS}^{+}$cells in the transplanted grafts compared with animals not performing exercise (Mann-Whitney $U$ test; $P=.02$ ). D, Gene expression of eNOS in aortic grafts as detected by polymerase chain reaction in the control group not receiving aorta transplantation. No significant difference was detectable between animals undergoing physical exercise or not (Mann-Whitney $U$ test; not significant). E, Gene expression of eNOS in aortic grafts as detected by polymerase chain reaction in the major alloantigeneic mismatch group did not reveal statistically significant between animals undergoing physical exercise or not (Mann-Whitney $U$ test; not significant). F, Gene expression of eNOS in aortic grafts as detected by polymerase chain reaction in the minor alloantigeneic mismatch group did not reveal statistically significant between animals undergoing physical exercise or not (Mann-Whitney $U$ test; not significant). n.s., Not significant.

performing regular physical exercise. These findings are suggestive of nitric oxide playing a pivotal role in the development of allograft vasculopathy and physical exercise being a regulative factor in this scenario. In this context, physical exercise leads to an upregulation of endothelial metabolism, because it similarly leads to an adjustment in glucose metabolism. ${ }^{23}$

In line with our results, other authors have shown that aerobic exercise not only increases the activity of eNOS, but also leads to an elevation of circulating endothelial progenitor cells (EPCs). ${ }^{24,25}$ In a study investigating the role of EPCs in the development of CAV, patients developing $\mathrm{CAV}$ revealed lower frequencies of circulating EPCs, potentially due to local recruitment of EPCs to areas of endothelial injury. ${ }^{26}$ Strikingly, in our study mice undergoing treadmill training revealed higher frequencies of $\mathrm{CD} 31^{+}$circulating endothelial cells.

Therefore, pathomechanisms similar to those occurring in the development of normal arteriosclerosis potentially also contribute to transplant vasculopathy. In summary, physical exercise could possibly recruit functional endothelial cells by elevating the number of EPCs. Simultaneously eNOS elevation leads to an improved availability of nitric oxide, with all of these mechanisms finally protecting graft endothelial function.

Still, although transplant arteriosclerosis occurred in our experimental model of fully allogeneic murine aorta transplantation without administration of immunosuppressive drugs, there is clear evidence for physical exercise being an important regulator for the degree of transplant arteriosclerosis. Therefore, including physical exercise in regular posttransplant care of heart transplant recipients could potentially reduce the risk of severe transplant vasculopathy and therefore prolong graft survival.

\section{CONCLUSIONS}

Physical exercise reduces the development of transplant atherosclerosis, potentially by augmenting endothelial cell turnover. This augmented turnover then counteracts endothelial dysfunction. Physical exercise should be part of the routine after heart transplantation.

\section{References}

1. ISHLT registry. J Heart Lung Transplant. 2012;31:1045-95. 
2. Rahmani M, Cruz RP, Granville DJ, McManus BM. Allograft vasculopathy versus atherosclerosis. Circ Res. 2006;99:801-15.

3. Hiemann NE, Wellnhofer E, Knosalla C, Lehmkuhl HB, Stein J, Hetzer R, et al. Prognostic impact of microvasculopathy on survival after heart transplantation: evidence from 9713 endomyocardial biopsies. Circulation. 2007;116:1274-82.

4. Kobashigawa JA, Katznelson S, Laks H, Johnson JA, Yeatman L, Wang XM, et al. Effect of pravastatin on outcomes after cardiac transplantation. $N$ Engl $J$ Med. 1995;333:621-7.

5. Fateh-Moghadam S, Bocksch W, Wessely R, Jager G, Hetzer R, Gawaz M. Cytomegalovirus infection status predicts progression of heart-transplant vasculopathy. Transplantation. 2003;76:1470-4.

6. Tona F, Marra MP, Fedrigo M, Famoso G, Bellu R, Thiene G, et al. Recent developments on coronary microvasculopathy after heart transplantation: a new target in the therapy of cardiac allograft vasculopathy. Curr Vasc Pharmacol. 2012;10:206-15.

7. Charles M, Charifi N, Verney J, Pichot V, Feasson L, Costes F, et al. Effect of endurance training on muscle microvascular filtration capacity and vascular bed morphometry in the elderly. Acta Physiol (Oxf). 2006;187:399-406.

8. Pingel J, Harrison A, Suetta C, Simonsen L, Langberg H, Bülow J. The acute effects of exercise on the microvascular volume of Achilles tendons in healthy young subjects. Clin Physiol Funct Imaging. 2013;33:252-7.

9. Hermann TS, Dall CH, Christensen SB, Goetze JP, Prescott E, Gustafsson F. Effect of high intensity exercise on peak oxygen uptake and endothelial function in long-term heart transplant recipients. Am J Transplant. 2011;11:536-41.

10. Institute of Laboratory Animal Resources, National Research Council. Principles of laboratory animal care, and the guide for the care and use of laboratory animals. Washington, DC: National Academies Press; 1996.

11. Koulack J, McAlister VC, Giacomantonio CA, Bitter-Suermann H, MacDonald AS, Lee TD. Development of a mouse aortic transplant model of chronic rejection. Microsurgery. 1995;16:110-3.

12. Theophile K, Jonigk D, Kreipe H, Bock O. Amplification of mRNA from lasermicrodissected single or clustered cells in formalin fixed and paraffin embedded tissues for application in quantitative real-time PCR. Diagn Mol Pathol. 2008;17: $101-6$.
13. Conti AA, Macchi C. Protective effects of regular physical activity on human vascular system. Clin Ter. 2013;164:293-4

14. Barton M. Prevention and endothelial therapy of coronary artery disease. Curr Opin Pharmacol. 2013;13:226-41.

15. Wienbergen $\mathrm{H}$, Hambrecht R. Physical exercise and its effects on coronary artery disease. Curr Opin Pharmacol. 2013;13:218-25.

16. Hsieh PL, Wu YT, Chao WJ. Effects of exercise training in heart transplant recipients: a meta-analysis. Cardiology. 2011;120:27-35.

17. Squires RW. Exercise therapy for cardiac transplant recipients. Prog Cardiovasc Dis. 2011;53:429-36.

18. Nytrøen KL, Rustad LA, Erikstad I, Aukrust P, Ueland T, Lekva T, et al. Effect of high-intensity interval training on progression of cardiac allograft vasculopathy. J Heart Lung Transplant. 2013;32:1073-80.

19. Colvin-Adams MI, Harcourt N, Duprez D. Endothelial dysfunction and cardiac allograft vasculopathy. J Cardiovasc Transplant Res. 2013;6: 263-77.

20. Billingham ME. Histopathology of graft coronary disease. J Heart Lung Transplant. 1992;11(3 Pt 2):S38-44.

21. Pethig KI, Heublein B, Wahlers T, Haverich A. Mechanism of luminal narrowing in cardiac allograft vasculopathy: inadequate vascular remodeling rather than intimal hyperplasia is the major predictor of coronary artery stenosis. Working Group on Cardiac Allograft Vasculopathy. Am Heart J. 1998;135 628-33.

22. Colvin-Adams M, Harcourt N, Duprez D. endothelial dysfunction and cardiac allograft vasculopathy. J Cardiovas Trans Res. 2013;6:263-77.

23. Swift DL, Lavie CJ, Johannsen NM, Arena R, Earnest CP, O'Keefe JH, et al Physical activity, cardiorespiratory fitness, and exercise training in primary and secondary coronary prevention. Circ J. 2013;77:281-92.

24. Perez-Terzic CM. Exercise in cardiovascular diseases. PM R. 2012;4:867-73.

25. Yetik-Anacak G, Catravas J. Nitric oxide and the endothelium: history and impact on cardiovascular disease. Vasc Pharm. 2006;45:268-76.

26. Liu Y, Li J, Zhang Z, Tang Y, Chen Z, Wang Z. Effects of exercise intervention on vascular endothelium function of patients with impaired glucose tolerance during prediabetes mellitus. Exp Ther Med. 2013;5:1559-65.

\title{
EDITORIAL COMMENTARY
}

\section{Of mice and men ... does exercise decrease progression of transplant coronary vasculopathy?}

\author{
William M. DeCampli, MD, PhD
}

See related article on pages 330-7.

In this issue of the Journal, Sommer and colleagues from the Hannover Medical School present the results of a well-designed study demonstrating reduced intimal

From The Heart Center at Arnold Palmer Hospital for Children, Orlando, Fla; and the College of Medicine, University of Central Florida, Orlando, Fla.

Disclosures: Author has nothing to disclose with regard to commercial support.

Received for publication Oct 23, 2014; accepted for publication Oct 25, 2014.

Address for reprints: William M. DeCampli, MD, PhD, Division of Cardiothoracic

Surgery, The Heart Center at Arnold Palmer Hospital for Children, 92 W Miller

St, Orlando, FL 32806 (E-mail: William.decampli@orlandohealth.com or

William.decampli@ucf.edu).

J Thorac Cardiovasc Surg 2015;149:337-9

$0022-5223 / \$ 36.00$

Copyright (c) 2015 by The American Association for Thoracic Surgery

http://dx.doi.org/10.1016/j.jtcvs.2014.10.107 proliferation after allogeneic transplant of a segment of abdominal aorta in mice thereafter subjected to 28 days of an exercise program, as compared with a group not subjected to exercise. The exercise group also demonstrated increases in endothelial integrity, circulating endothelial cells (ECs), and EC function as measured by counts of ECs staining positive for the anti-endothelial nitric oxide synthase antibody ab5589. The results are not subtle. The percentages of luminal area reduction were $81 \%$ in the control group and $60 \%$ in the exercise group. In major histocompatibility complex-matched groups (ie, only minor mismatch present), the reductions were $75 \%$ in the control group and $49 \%$ in the exercise group.

In general, exercise is known to improve metabolic function, lipid profile (particularly, increase in highdensity lipoprotein cholesterol), insulin sensitivity, and 